\title{
KINETIC DATABASE FOR ASTROCHEMISTRY
}

\author{
V. Wakelam ${ }^{1}$ and the KIDA team
}

\begin{abstract}
KIDA (for KInetic Database for Astrochemistry) is a project initiated by different communities in order to 1) improve the interaction between astrochemists and physico-chemists and 2) simplify the work of modeling the chemistry of astrophysical environments. KIDA was designed to group at the same place and with the same format all gas-phase chemical reactions useful for astrochemical simulations of the interstellar medium and planetary atmospheres. From KIDA, a subset of chemical reactions has been extracted for the cold and dense interstellar medium: kida.uva.2001.
\end{abstract}

\section{Introduction}

Modeling of chemical processes in the interstellar medium and in planetary atmospheres (hereafter referred to as astrophysical environments) share common difficulties, namely a lack of reference data for many processes and large uncertainty for those data which have been measured in the laboratory, in physical conditions generally not representative of the space environment. A permanent link between modelers and physico-chemists appears therefore as a necessity for modelers to publicize their most urgent data needs and for physico-chemists to advertise new data. This is the best way to prevent the use of inappropriate or obsolete data/extrapolations in chemical networks. KIDA is a project initiated by different communities in order to i) improve the interaction between astrochemists and physico-chemists and ii) simplify the work of modeling the chemistry of astrophysical environments.

\section{The database}

The KIDA database (http: //kida.obs.u-bordeaux1.fr) is extensively described in Wakelam et al. (2012). We summarize here a few important aspects of the

${ }^{1}$ Université Bordeaux, LAB, UMR 5804, 33270 Floirac, France and CNRS, LAB, UMR 5804, 33270 Floirac, France 


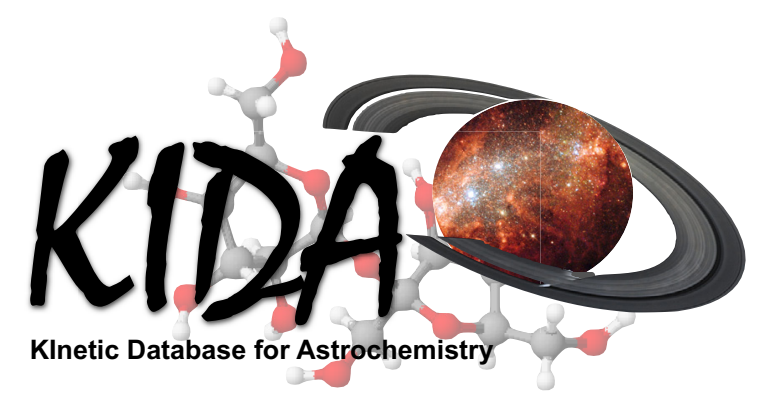

Fig. 1. KIDA logo.

database and its web interface. The database provides the user with extensive information about the data (references, details on the methods to obtain the data, validity range of temperature, etc.). The interface enables users to attach comments or new information to data already stored in KIDA, or to populate the database with new data. The reviewing of new data by a group of experts prior to publication in the database is a strong asset of KIDA. The role of the KIDA experts is to validate the addition of data from data providers and give recommendation about the rate coefficients to use in specific physical conditions. In case several rate coefficients exist for the same reaction and range of temperature based on different experiments or calculations, new studies can be done or the experts defines an uncertainty range taking into account the different results. In most cases, when a recommendation is given, the details of the expertise can be seen in a data sheet provided on the reaction page. Recommendations can be of four types: (1) not recommended; (2) not rated; (3) valid; and (4) recommended value. Modelers can extract lists of reactions from KIDA, based on different search criteria. The lists are automatically commented to alert the user on reactions that might be problematic.

All the reactions from the low-temperature OSU database osu-01-2009 have been included in KIDA, even reactions involving anionic species (Harada \& Herbst 2008). Many updates concerning photoinduced rates are currently being undertaken based on results from van Dishoeck et al. (2006) and van Hemert \& van Dishoeck (2008). Branching ratios for the dissociation of carbon clusters by both external UV radiation and cosmic ray-induced photons have been added based on Chabot et al. (2010). Reactions necessary for primordial chemistry, collected by D. Galli, will be included before the end of 2012. Finally, many other additions have been made, in particular for neutral-neutral reactions, based on new experimental and/or theoretical results. KIDA will soon contain kinetic data for planetary atmospheres (other than the Earth). The quality of the data present in KIDA will be improved over the years by KIDA experts and will be preserved thanks to the detailed information stored in KIDA. The visibility of KIDA will be improved by its interoperability with the Virtual Atomic and Molecular DataCenter (Dubernet et al. 2010, http://www.vamdc.eu/) and the Europlanet Research Infrastructure (http://www. europlanet-eu.org/outreach/). 


\section{Application}
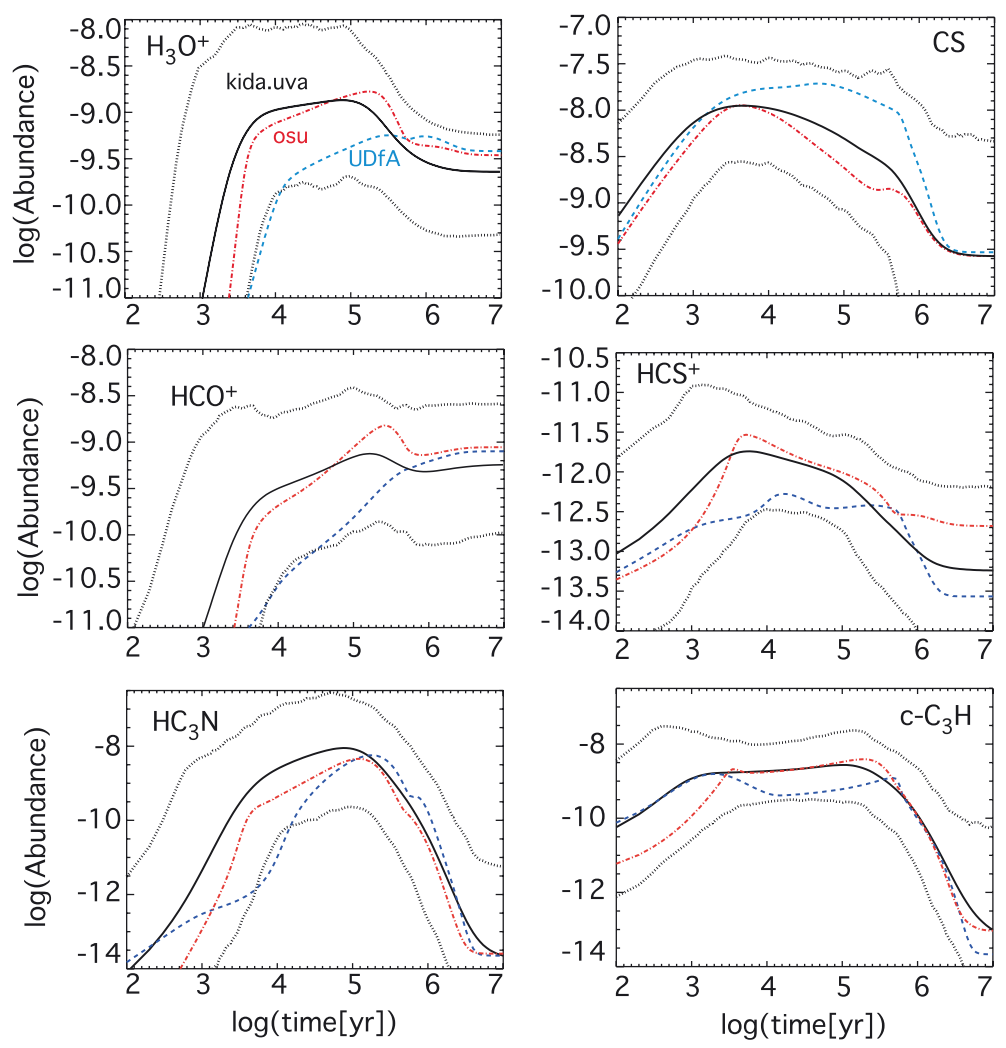

Fig. 2. Abundances as a function of time for selected species computed for interstellar dense cloud conditions (gas temperature of $10 \mathrm{~K}, \mathrm{H}_{2}$ density of $2 \times 10^{4} \mathrm{~cm}^{-3}$, Av of 30 and cosmic-ray ionization rate of $1.3 \times 10^{-17} \mathrm{~s}^{-1}$ ) using three different chemical networks: udfa06 (blue dashed lines), osu-01-2009 (red dashed-dotted lines) and kida.uva.2011 (black lines). Dotted curves represent $2 \sigma$ error bars on the abundances computed with the kida.uva.2011 network using uncertainty propagation in the reaction rate coefficients. The figure is taken from Wakelam et al. (2012).

KIDA contains reaction rate coefficients for various types of environments, i.e. various temperature and density ranges. Since it is not necessarily easy for users to select a correct sublist of reactions for a specific application, we provide one for cold (10 to $300 \mathrm{~K}$ ) and dense (above a few $10^{4} \mathrm{~cm}^{-3}$ ) interstellar environments. This network is called kida.uva.2011 (http://kida.obs.u-bordeaux1. $\mathrm{fr} /$ models) and will be updated every year based on the data included in KIDA. We show in Figure 2 an application of this network, compared to other public networks (udfa06, http://www.udfa.net/ and osu-01-2009 http://www.physics. ohio-state.edu/ eric/research.html). 
Species shown in the figure have been selected because of the differences obtained with the different networks. Abundances obtained with the OSU and kida.uva networks are similar because KIDA is mainly based on the OSU compilation with a few of updates. Abundances computed with udfa06 on the contrary can be different, as shown especially for CS. Model predictions done with the different networks are however within the $2 \sigma$ error bars computed from the uncertainty propagation of rate coefficients, which are typically an order-of-magnitude in both directions.

The KIDA team acknowledges various sources of funding, which have allowed us to develop this database. These sources include The University of Bordeaux, The "Institut de Physique Fondamentale de Bordeaux", the CNRS/INSU (PCMI, PNP and ASOV), The Observatoire Aquitain des Sciences de l'Univers. We acknowledge support from the European Research Council (ERC Grant 209622: E ${ }_{3}$ ARTHs), the Agence Nationale de la Recherche (ANR-JC08_311018: EMA:INC), the European program Astronet (CATS project), and the European Seventh Framework Programme (FP7: VAMDC and EUROPLANET projects). VAMDC is funded under the Combination of Collaborative Projects and Coordination and Support Actions Funding Scheme of The Seventh Framework Program (Call topic: INFRA-2008-1.2.2 , Grant Agreement number: 239108). The EUROPLANET RI (Research Infrastructure) is funded under the Grant Agreement number: 228319.

\section{References}

Chabot, M., Tuna, T., Béroff, K., et al., 2010, A\&A, 524, 39

van Dishoeck, E.F., Jonkheid, B., \& van Hemert, M.C., 2006, Faraday Discussions', 133, 231

Dubernet, M.-L., Boudon, V., Culhane, J.L., et al., 2010, J. Quant. Spectr. Rad. Transfer, 111,2151

Harada, N., \& Herbst, E., 2008, ApJ, 685, 272

van Hemert, M.C., \& van Dishoeck, E.F., 2008, Chem. Phys., 343, 292

Wakelam, V., Herbst, E., Loison, J.-C., et al., 2012, ApJS, 199, 21 